\title{
Parallel Surveys of Specialists and Family Physicians in Nova Scotia Regarding Satisfaction With the Referral Process
}

\author{
Blair A. Williams', Peter A.M. Anderson² \\ ${ }^{1}$ Class of 2011, Faculty of Medicine, Dalhousie University \\ ${ }^{2}$ Division of Urology, Department of Surgery, Dalhousie University, Halifax, Nova Scotia, Canada
}

\begin{abstract}
Background: The referral letter is fundamental to effective communication between family physicians (FPs) and specialists. However, this document is frequently cited as a source of frustration for both referring and consultant physicians.

Methods: Aspects of the referral process were identified and assembled in 2 surveys: one distributed to FPs and one to specialists. The survey used a 5-point Likert scale for each aspect of the referral process. Surveys were mailed to 500 family physicians and 500 specialists.

Results: There was a $42.4 \%$ and $43.8 \%$ response rate from FPs and specialists, respectively. Few of the survey items stood out as being particularly polarizing. There was no significant difference between FPs and specialists in the level of satisfaction, nor among the groups of specialists. Among FPs, those practicing outside of the Capital District Health Authority (CDHA) had significantly higher overall satisfaction than those practicing within the CDHA.

Conclusion: The results of the survey indicate that the referral letter could be improved by focusing on specific aspects of the letter. These points can be targeted at all levels of medical education (undergraduate, postgraduate, and continuing medical education) to help improve a process that is fundamental to our healthcare system. The disparity in overall satisfaction between FPs in CDHA and FPs in the rest of the province is intriguing given that CDHA has the greatest number of specialists.
\end{abstract}

\section{Introduction}

Referrals from family physicians (FPs) to specialist physicians are a cornerstone of the Canadian healthcare system. Replies from the specialists to the FPs are an important opportunity to relay information to the FP, to communicate a treatment regimen, and to build professional relationships. Effective referrals require that referring physicians have a certain level of knowledge of the specialty to which they refer. This facilitates communication of the patient's medical issue and allows for the formulation of a clear question for the consultant. Likewise, the reply letter should include pertinent information to allow the FP to continue to care for the patient.

Dissatisfaction with the referral process has been a recurrent theme in Canadian healthcare ${ }^{1,2}$. The 2007 Canadian National Physician Survey found that only $24.1 \%$ of FPs and $13.8 \%$ of specialists replied 'Very Satisfied' when asked to rate the usefulness and reliability of the referral process ${ }^{3}$. With respect to relationships with specialists, only $23.7 \%$ of FPs were 'Very Satisfied, verifying that there is room for improvement in our current system.

One possible explanation for the current dissatisfaction with the referral process centers on the change in Canadian post-graduate medical training in 1991 (i.e., the discontinuation of the rotating internship). The decreased exposure to specialties during Family Medicine residency may have resulted in a perceived decrease in general practitioner expertise and increase in unnecessary referrals. Likewise, it may be perceived that fewer specialists begin their careers in general practice and so lack that perspective, leading to deficient reply letters.

Studies of interventions to improve communication between FPs and specialists suggest that education repeatedly yields positive results with respect to the quality of referrals ${ }^{4,5}$. It remains unclear which aspects 
of the referral process are most in need of improvement and thus should be targeted by education efforts.

In order to further study the sources of frustration with the referral process we undertook to assess the level of satisfaction with the process among NS physicians. Specific aspects of the referral letter were identified and NS specialists were surveyed regarding their satisfaction with these items and with the referral process as a whole. Information was also collected on the year the physician graduated from medical school, their specialty, and in which district health authority they practice. We also surveyed FPs in Nova Scotia, identifying specific aspects of the reply letter and gauging their satisfaction. Year of graduation from medical school and district health authority information were also collected. Comments on the referral system were also solicited from both specialists and FPs.

\section{Material and Methods}

A questionnaire was developed to gauge the level of satisfaction with the referral process in Nova Scotia. Two different, though similar, surveys were created for distribution to specialists and to family physicians. The surveys consisted of 13 questions related to the referral letter, adapted from Keely et $\mathrm{al}^{6}$ and several other questions related to the district health authority in which the physicians worked, and their length of time in the profession. Opportunity was also provided to comment on subject matter not covered by the survey. The questions related to referral content were to be answered on a 5-point Likert scale: 1 'Very Unsatisfied', 2 'Somewhat Unsatisfied', 3 'Neutral', 4 'Somewhat Satisfied', and 5 'Very Satisfied.

Both surveys were validated by distribution of the specialist survey to 10 specialists and the FP survey to 10 family physicians, along with a cover letter explaining the goals of the project. Feedback was collected and integrated into the final version of the questionnaires (Appendix). The results of the surveys circulated for validation were not included in the final analysis.

There were 1192 specialists and 1121 FPs registered with the College of Physicians and Surgeons of Nova Scotia (CPSNS), as of August 2008. Surveys were distributed by mail to 500 specialists and 500 FPs (randomly selected and licensed to practice in Nova Scotia), along with a cover letter explaining the purpose of the study. The mailing addresses were obtained through the CPSNS. Return envelopes with postage were included to ensure anonymity of returned surveys.
Data from the returned surveys was analyzed using SPSS statistics software (Chicago, Illinois). The frequencies of each response were calculated and the relationship between geographic region and the responses was determined with an independent samples t-test. For the purposes of analyzing the relationship between time in practice and overall satisfaction, we chose a cut-off of medical school graduation year 1991 to create two groups, allowing for the use of an independent samples t-test for analysis. The year 1991 was chosen because it was the last year in which one could enter a rotating internship before its abolition. The relationship between the individual specialty and satisfaction with the referral process was also examined. Specialties were grouped in 4 groups: psychiatry, internal medicine, pediatrics, and surgery. Certain specialties were excluded prior to distribution based on the lack of traditional referrals (e.g., pathology, diagnostic imaging). This aspect was analyzed using a one-way ANOVA. Written-in comments were reviewed and recurrent themes have been included in the discussion of this paper. Statistical results are reported as mean response on the 5-point scale $+/$ - standard error of the mean. Significance was determined using a 95\% confidence interval.

\section{Results}

Specialist Survey

A response rate of $42.4 \%$ was attained in the specialist survey, yielding a margin of error of $3.39 \%$ for reported frequencies. $72.3 \%$ of responding specialists were from the CDHA and $27.7 \%$ from outside the CDHA. With respect to category of specialty, $12.0 \%$ of respondents were from psychiatry, $43.5 \%$ from internal medicine, $16.7 \%$ from pediatrics, and $27.8 \%$ from surgery. The distribution of these specialties in NS, according to

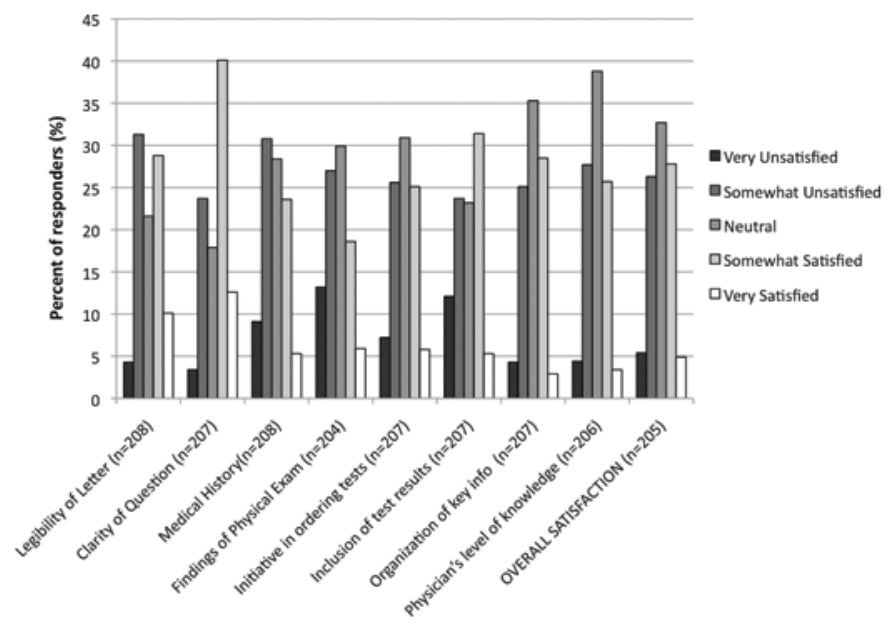

Figure 1. Frequencies of response (\%) for each item on the survey completed by specialists. 


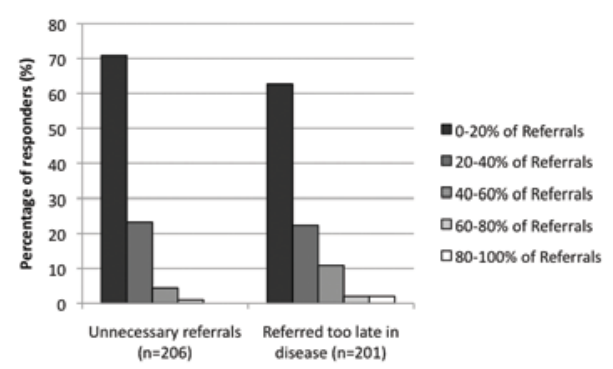

Figure 2. Frequencies of responses (\%) to questions regarding timing and necessity of referrals.

statistics released by the Canadian Medical Association, is $17.2 \%$ in psychiatry, $35.7 \%$ in internal medicine, $9.6 \%$ in paediatrics, and $37.2 \%$ in surgery ${ }^{7}$.

In rating the length of the referral letter, $49.4 \%$ of specialists felt it was too short, $50.6 \%$ said it was an appropriate length, and no specialists felt that the referral letter was too long. The frequencies of responses for the survey of specialists are presented in Figure 1. Most items tended toward neutral. Items with which responding specialists were dissatisfied include medical History, physical exam, and FP's level of knowledge.

Specialists were further asked to rate what percentage of referrals, on average, are unnecessary and how many come too late in the disease process. The frequencies of their replies are presented in Figure 2. The majority of specialists felt that $0-20 \%$ of referrals are unnecessary and that $0-20 \%$ of referrals come too late in the disease process.

In statistical analysis, there was no significant difference in overall satisfaction between specialists who graduated from medical school before $(2.91+/$ $0.095 ; \mathrm{n}=139)$ or after $(2.90+/-0.133, \mathrm{n}=67)$ the 1991 change in postgraduate training $(\mathrm{P}=0.91)$. There was no significant difference $(\mathrm{P}=0.96)$ in average overall satisfaction between specialists practicing in the CDHA $(2.90+/-0.094 ; \mathrm{n}=148)$ and those practising outside $(2.89+/-0.139 ; \mathrm{n}=58)$ CDHA. There were no significant differences among any of the specialty categories in average ratings of overall satisfaction ( $\mathrm{P}>0.05$ for all combinations). In comparing average ratings of overall satisfaction between the FP survey $(2.87+/-0.076 ; \mathrm{n}=218)$ and the specialist survey $(2.90$ $+/$ - 0.077), there was no significant difference $(\mathrm{P}=0.07)$.

\section{Family Physician Survey}

The survey distributed to FPs to assess their satisfaction with the reply letter attained a total response rate of
$43.8 \%+/-3.24$. The distribution of respondents was 53.4\% from the Capital District Health Authority (CDHA) and $46.6 \%$ from outside of the CDHA.

None of the FPs who responded felt that the reply letter was too short, $97.7 \%$ felt that the letter was appropriate in length, and $2.3 \%$ felt that it was too long. The frequencies of replies regarding specific aspects of the reply letter included in the survey are presented in Figure 3. In general, FPs were satisfied with the aspects of the reply letter identified in the survey. The aspects of the referral process with which FPs seem to be dissatisfied are Timeliness of consult and Availability of specialists. Overall satisfaction with the referral process was rated on the dissatisfied end of the scale.

Whether the FP graduated from medical school before $(2.83+/-0.097 ; \mathrm{n}=150)$ or after $(2.92+/-0.123 ; \mathrm{n}=64)$ the 1991 change in postgraduate training did not have a significant effect on overall satisfaction with the referral process $(\mathrm{P}=0.573)$.

Mean rankings of overall satisfaction by FPs from outside of the CDHA $(3.23+/-0.112 ; n=101)$ compared to FPs practicing in the CDHA $(2.52+/-0.098 ; n=111)$ were significantly different $(\mathrm{P}=0.0001)$, with FPs practicing outside the CDHA being more satisfied with the referral process.

\section{Discussion}

The high response rates attained in both surveys speaks to the importance of this issue to Nova Scotia physicians. Both response rates were considerably higher than the 2007 National Physician Survey, which attained a response rate of $36.9 \%$ from NS physicians ${ }^{3}$. This is an excellent response rate for survey research, particularly among health care professionals.

There were no aspects of the referral letter that were particularly polarizing among specialists, with median responses being either neutral, or somewhat satisfied/ dissatisfied (Fig. 1). Aspects of the referral letter that were rated on average unsatisfactorily included legibility of letter, inclusion of medical history/physical exam, and physician's level of knowledge. Satisfactory aspects included clarity of question, inclusion of test results, organization, and overall satisfaction. The vast majority of specialists felt that the referrals were appropriate and that referrals came at the appropriate point in the disease process (Fig. 2).

These findings give clear direction that inclusion of relevant history and physical exam are areas for improvement in the referral letter. With the growing 


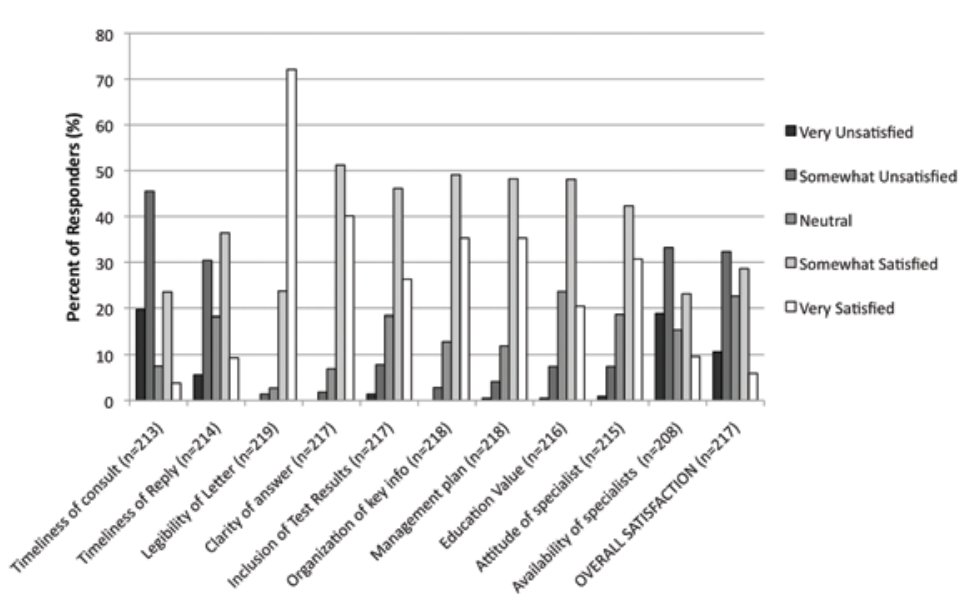

Figure 3. Frequency of response (\%) for each item on the survey completed by family physicians.

use of electronic medical records in Nova Scotia, legibility issues should decrease over time. The rating of physician's level of knowledge being rated on average unsatisfactory (median rating neutral) is a more difficult issue to address and would require change in under- and post-graduate training programs. Further study in this area is needed to determine which specialties are particularly unsatisfied, though there is no significant difference among the four subgroups of specialists identified in this survey. Furthermore, statistics comparing location of practice and timing of graduation with overall satisfaction with the referral process revealed no significant differences. There was also no significant difference in overall satisfaction between FPs and specialists.

Results from the survey distributed to FPs regarding satisfaction with the reply letters were favorable with respect to individual aspects of the letter (Fig. 3). The unfavorable responses regarded availability of specialists and the timeliness of consultations. Despite the fact that most aspects of the reply letter were rated high, overall satisfaction with the referral process was rated low. Clearly, satisfaction with individual aspects of the reply letter is insufficient to confer satisfaction with the referral process as a whole, presumably due to dissatisfaction regarding availability and timeliness.

The only significant association in the analyses of these surveys was with regard to the location of practice of FPs. Compared to those in the rest of the province, FPs within the CDHA had significantly lower mean responses to overall satisfaction with the referral process. This could be due to the fact that the specialists' time is divided between patient care, medical education, and research. However, further study into the explanation of the regional difference in satisfaction is needed.

A frequent theme of written comments on the returned surveys from both FPs and specialists was the extreme variability in experiences with referrals. Responders stated that this made it difficult to give a general ranking of the survey items since they have had experiences across the spectrum. FPs cited frustration with lack of acknowledging receipt of referral letters by specialists, and 'downloading' of work from specialists to FPs. Specialists called for more typed referrals since handwritten notes are often illegible. Suggestions for improvements included standardized forms, electronic referral system, and a system to log wait times. These are timely comments since the College of Physicians and Surgeons of Nova Scotia has recently developed Guidelines for Physicians Regarding Referral and Consultation which is very much in line with the results of this survey ${ }^{8}$.

The high response rates attained in both surveys speaks to the importance of this issue to Nova Scotia physicians. Both response rates were considerably higher than the 2007 National Physician Survey, which attained a response rate of $36.9 \%$ from NS physicians ${ }^{3}$. Though the results do not reveal any staggering deficits in the referral process, there is clearly room for improvement. A neutral rating should not be considered adequate. Medical education programs at all levels can focus on encouraging the inclusion of medical history and physical exam findings. Electronic medical records and the growing use of standardized referral forms should help improve legibility of issues. FPs would appreciate acknowledgement of receipt of referrals by specialists, with an estimate of wait times. Availability of specialists is a systemic problem that was identified as a major source of frustration but is beyond the scope of this study. 


\section{Appendix}

\section{Specialist Survey}

What is your speciality?

In what year did you graduate from medical school?

In which District Health Authority do you practice?

In general, how satisfied are you with each of the following aspects of the referrals that you receive? (please select one response for each question)

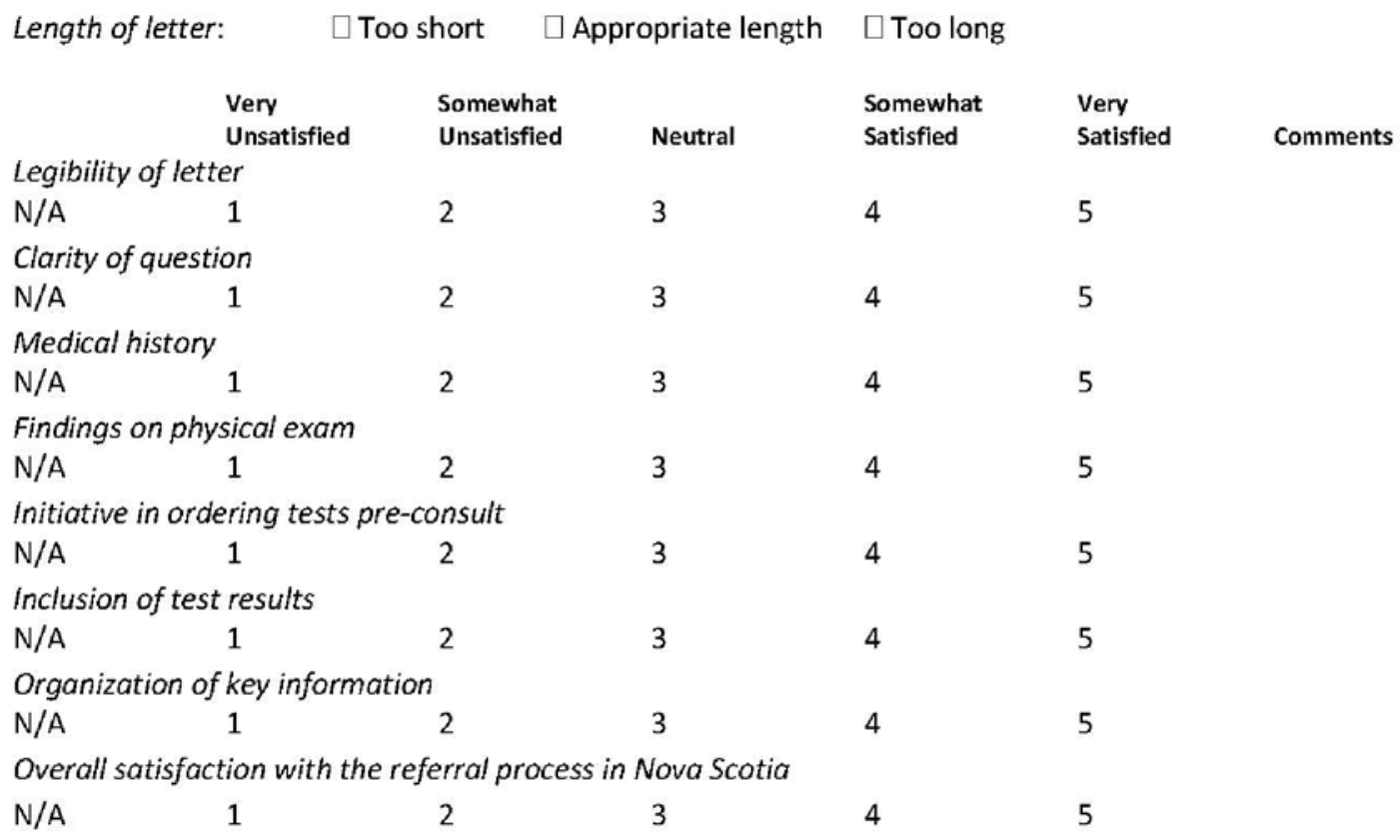

What percentage of referrals/consults do you judge to be frivolous or unnecessary?
$0-20 \%$
$20-40 \%$
$\square \quad 40-60 \%$
口 $60-80 \%$
$80-100 \%$

What percentage of referrals/consults do you judge to come to you too late in the disease process?
$0-20 \%$
$\square \quad 20-40 \%$
口 $40-60 \%$
$\square \quad 60-80 \%$
. $80-100 \%$

How satisfied are you with the referring physician's level of knowledge, as it pertains to your speciality?

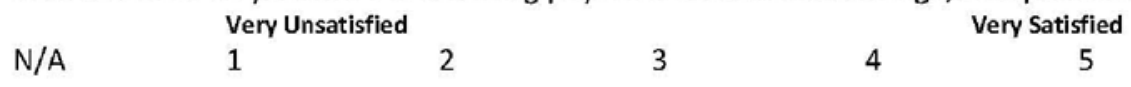

If unsatisfied with the level of knowledge, which group do you feel has the least knowledge of your speciality:
Family Physicians
Other Specialists
No Difference

Other comments: 


\section{Appendix}

\section{Family Physician Survey}

In what year did you graduate from medical school?

In which District Health Authority do you primarily practice?

How satisfied are you with each of the following aspects of the consultation reports that you receive from specialists? (please select one response for each question)

Length of letter: $\quad \square$ Too short $\quad \square$ Appropriate length $\quad \square$ Too long

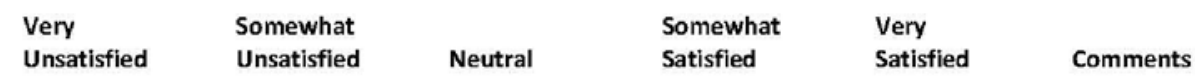

Timeliness of consultation

Timeliness of reply

N/A $\quad 1$

Legibility of letter

N/A $\quad 1$

2

Clarity of answer

N/A $\quad 1$

Inclusion of test results

N/A $\quad 1$

Organization of key information

N/A $\quad 1$

Management plan

N/A $\quad 1$

Educational value

N/A $\quad 1$

Attitude of specialist

$\begin{array}{llllll}\text { N/A } & 1 & 2 & 3 & 4 & 5\end{array}$

Availability of specialists for consultation

$\begin{array}{llllll}\text { N/A } & 1 & 2 & 3 & 4 & 5\end{array}$

Overall satisfaction with the referral process in Nova Scotia
N/A
1
2
3
4
5

What percentage of consultation reports do you feel come back too late in the disease process to be of benefit to the patient?
口 $0-20 \%$
口 $20-40 \%$
$40-60 \%$
$60-80 \%$
$80-100 \%$

Other comments: 
